\title{
Influences of Diabetes on Hearing Recovery in Noise-Exposed Mice
}

\author{
Chan Joo Yang ${ }^{1}$, Ji-Won Lee ${ }^{2}$, and Jong Woo Chung ${ }^{1}$ \\ ${ }^{1}$ Department of Otolaryngology, ${ }^{2}$ Asan Institute for Life Science, Asan Medical Center, University of Ulsan College of Medicine, \\ Seoul, Korea
}

$\begin{array}{ll}\text { Received } & \text { September 26, } 2015 \\ \text { Revised } & \text { November 5, 2015 }\end{array}$

Accepted November 18, 2015

\section{Address for correspondence \\ Jong Woo Chung, MD, PhD \\ Department of Otolaryngology, \\ Asan Medical Center, \\ University of Ulsan \\ College of Medicine, \\ 88 Olympic-ro 43-gil, Songpa-gu, \\ Seoul 05505, Korea \\ Tel +82-2-3010-3718 \\ Fax +82-2-489-2773 \\ E-mail jwchung@amc.seoul.kr}

\begin{abstract}
Background and Objectives: Many studies have reported an association between diabetes and hearing loss. However, these reports were mainly abstractive correlations between common hearing loss and the incidence of diabetes. Therefore, we evaluated the impact of diabetes on the occurrence of and recovery from noise-induced hearing loss. Materials and Methods: We used 5-week-old C57BLKS/J-m wild type (+/+) and C57BLKS/J-db/db male mice as the control and diabetic groups, respectively. In one set of experiments, the hearing levels of control and diabetic mice were measured weekly for 7 weeks. In a second set of experiments, control and diabetic mice were exposed to broadband white noise of 110 dB SPL for 3 hours; hearing levels were analyzed before and immediately after exposure, 1, 3, and 5 days, and 1, 2, 3, and 4 weeks after the noise exposure. Results: The hearing levels of the control group were better than those of the diabetic group at each weekly revision for 7 weeks at all auditory brainstem response frequencies $(4,8,16$, and $32 \mathrm{kHz})$. After noise exposure, both groups of mice showed an immediate increase in the hearing level threshold at all frequencies. Subsequent threshold recovery was seen in both groups with no difference in the hearing level recovery rates between the two groups. Conclusions: Hearing level with aging becomes significantly impaired earlier in diabetic mice but hearing recovery after noise exposure is similar between diabetic and control mice.
\end{abstract}

J Audiol Otol 2015;19(3):138-143

KEY WORDS: Diabetes mellitus $\cdot$ Hearing loss $\cdot$ Noise-induced $\cdot$ Cochlea $\cdot$ Mice.

\section{Introduction}

Diabetes was recently recognized to induce microvascular complications affecting the renal, visual, and peripheral nervous systems $[1,2]$. The clinical manifestation of diabetes mellitus is characterized by vascular and neuropathic complications $[3,4]$. In the inner ear, angiopathy may occur both directly-by interfering with cochlear blood supply and reducing nutrient transportation-and indirectly-by reducing blood flow due to narrowed vasculature-causing secondary degeneration of the eighth cranial nerve [5].

Although many studies have reported an association between diabetes and hearing loss, these reports have had some

This is an Open Access article distributed under the terms of the Creative Commons Attribution Non-Commercial License (http://creativecommons. org/licenses/by-nc/3.0/) which permits unrestricted non-commercial use, distribution, and reproduction in any medium, provided the original work is properly cited. limitations; the use of data from animal studies, few subjects, or a cross-sectional study design [6,7]. Most studies have reported only an abstractive correlation between common hearing loss and the incidence of diabetes. Thus, there is a need for further research with experimental conditions that control for other variables beyond diabetes, such as noise-induced hearing loss. Accordingly, we conducted our present study to evaluate the impact of diabetes on the occurrence of and recovery from noise-induced hearing loss. We compared the sensitivity and the recovery for noise-induced hearing loss between normal and diabetic mice.

\section{Materials and Methods}

\section{Experimental animals}

We used 5-week-old male mice with a normal Preyer's reflex and normal hearing threshold; C57BLKS/J-m wild type 
$(+/+)$ mice were used as the control group (11 mice) while $\mathrm{C} 57 \mathrm{BLKS} / \mathrm{J}-\mathrm{db} / \mathrm{db}$ mice were used as the diabetic group (13 mice). The mice were allowed to adapt to the laboratory environment for 1 week before the experiment. The animals were housed in silent cages at a temperature of $24 \pm 1^{\circ} \mathrm{C}$ temperature and maintained in environmentally controlled rooms with a 12-h light/dark cycle, with food and water available ad libitum.

Zolazepam/tiletamine (Zoletil; Virbac, Carros, France) 25 $\mathrm{mg} / \mathrm{kg}$ and xylazine (Rompun; Bayer, Seoul, Korea) $10 \mathrm{mg} /$ $\mathrm{kg}$ were administered intraperitoneally for anesthesia, and half of the above amounts were additionially added if necessary. All animal experiments were performed with the approval of the Animal Care Committee of the University of Ulsan College of Medicine.

\section{Experimental protocol}

\section{Resting hearing threshold with aging}

Mice were divided into a control group (8 mice) and a diabetic group ( 9 mice). The hearing levels of each mouse, none of whom was exposed to white noise were evaluated weekly for 7 weeks.

\section{Hearing threshold changes after noise exposure}

The remaining animals were divided into two groups, a control group ( 3 mice) and a diabetic group (4 mice). Both control and diabetic groups were exposed to broad band white noise of $110 \mathrm{~dB}$ SPL (sound pressure level) in a soundproof booth for 3 hours. White noise $(0.3-10 \mathrm{kHz})$ was generated by a personal computer and amplifier (R-399; Interm, Seoul, Korea) and delivered through a speaker (290-8L; Altec Lansing, Oklahoma City, OK, USA) in a noise booth. The amplifier with the speaker on top was placed in the left corner of the noise booth, and the horn was attached at a 45-degree angle. The hearing levels of each mouse were analyzed before and immediately after noise exposure and at following periods; $1,3,5$, and 7 days, and 2, 3, and 4 weeks after exposure.

\section{Hearing measurements}

For the measurement of hearing levels, we analyzed the auditory brainstem response (ABR) and distortion product otoacoustic emission (DPOAE) results with an auditory evoked potential workstation (Tucker-Davis Technologies, Alachua, FL, USA). Tone-burst stimulation was used to measure frequency-specific ABRs. The frequencies measured were $4,8,16$, and $32 \mathrm{kHz}$, and we gained the waveform by repeatedly decreasing the stimulation tone by $10 \mathrm{~dB}$ from a

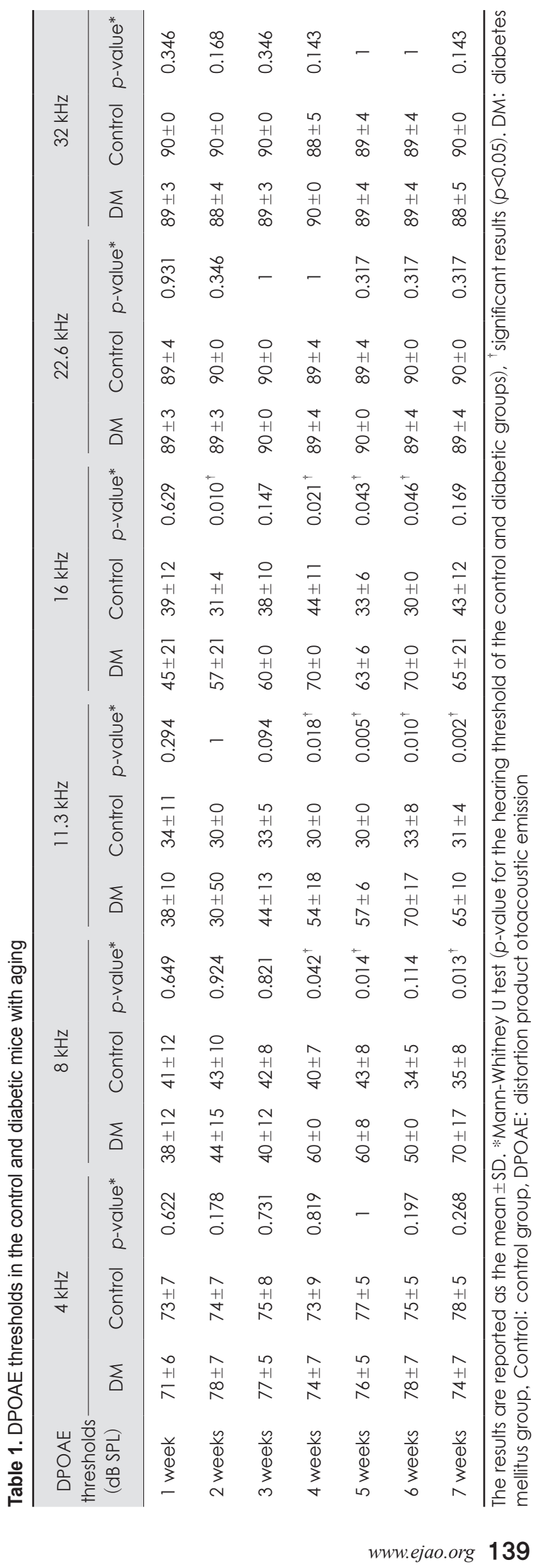


strength of $90 \mathrm{~dB}$ SPL. The most uniformly generated waveform was used for analysis. We judged a waveform with an amplitude greater than $0.2 \mu \mathrm{V}$ that appeared to be similar to the response to the previous stimulation tone to be a significant waveform. DPOAE was measured using an amplifier system that could provide two stimulation tones. We generated $\mathrm{f} 1$ and $\mathrm{f} 2$ primary stimulation tones $(\mathrm{f} 2 / \mathrm{f} 1=1.2)$ using a dual channel synthesizer. For the measurement of frequencyspecific responses, we set the $\mathrm{f} 2$ stimulation tone to 4,8 , $11.3,16,22.6$, and $32 \mathrm{kHz}$. The threshold was determined when the DPOAE response exceeded the noise level on the measured graph at each frequency.

\section{Statistical analysis}

The hearing thresholds of the control and diabetic groups were compared using the Mann-Whitney U test. To evaluate the effects of diabetic on recovery from noise-induced hearing loss, we calculated the rate of hearing level recovery each week. The recovery rate for each week was obtained as follows:

Recovery rate $=($ recovered hearing threshold/deteriorated hearing threshold) $\times 100 \%$

where the recovered hearing threshold is the difference in the hearing threshold immediately after noise exposure and at each week and the deteriorated hearing threshold is the difference in the hearing threshold before and immediately after noise exposure.

The hearing recovery rates of the two groups were statistically validated with the Mann-Whitney U test. Values of $p<0.05$ were considered significant. Hearing thresholds are shown as the mean \pm SD. All statistical analyses were performed using SPSS version 21.0 (IBM, Armonk, NY, USA).

\section{Results}

\section{Changes in resting hearing thresholds with aging}

The hearing levels of the control group were better than those of diabetic group from 1 to 7 weeks at all ABR frequencies $(4,8,16$, and $32 \mathrm{kHz})$. The average ABR thresholds at 1 week at $4,8,16$, and $32 \mathrm{kHz}$ were $44 \pm 5 \mathrm{~dB}$ SPL, $33 \pm 8$ $\mathrm{dB}$ SPL, $54 \pm 5 \mathrm{~dB}$ SPL, and $57 \pm 9 \mathrm{~dB}$ SPL, respectively, in the diabetic mice and $36 \pm 4 \mathrm{~dB}$ SPL, $26 \pm 8 \mathrm{~dB}$ SPL, $36 \pm 4$ $\mathrm{dB}$ SPL, and $34 \pm 5 \mathrm{~dB}$ SPL, respectively, in the control group. The ABR thresholds of the diabetic group were significantly poorer than those of the control group at all frequencies at 1,2, and 3 weeks, and the DPOAE thresholds of the diabetic group were significantly worse than those of the control group at 8 , 11.3, and $16 \mathrm{kHz}$ at 4 and 5 weeks (Table 1, Fig. 1).

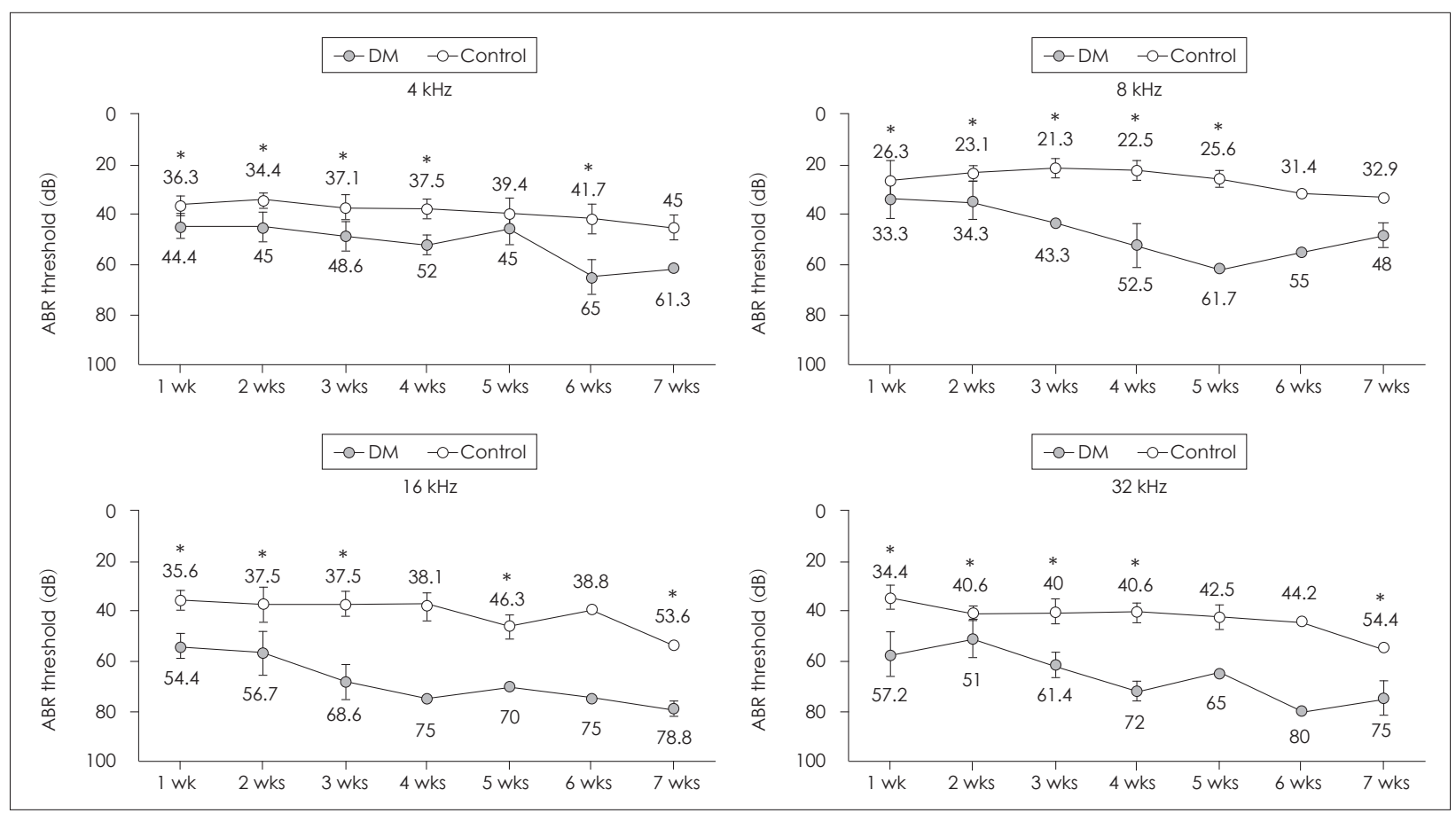

Fig. 1. Thresholds of the auditory brainstem response in control and diabetic mice with aging. The results are reported as the mean $\pm S D$. ${ }^{*} p<0.05$ ( $p$-value for the hearing threshold of the control and diabetic groups, Mann-Whitney U test). DM: diabetes mellitus group, Control: control group. 


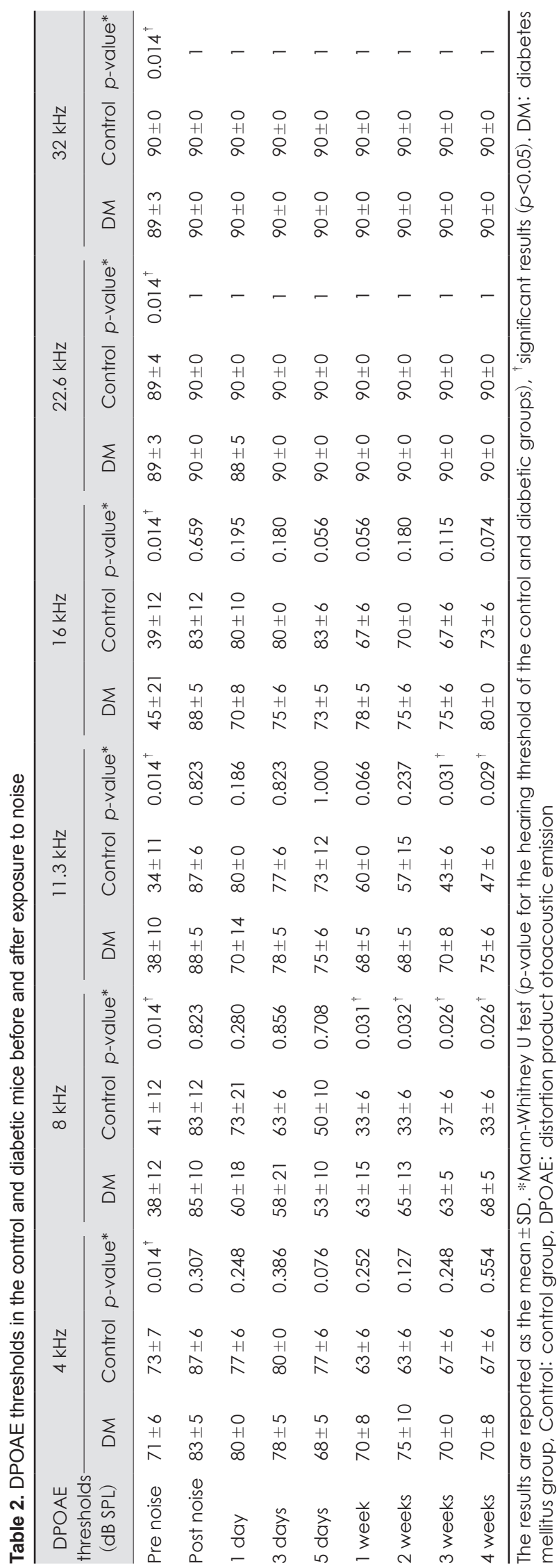

\section{Hearing thresholds changes after noise exposure}

Both the diabetic and control groups showed an increase in the hearing threshold immediately after noise exposure, which began to slowly recover at all frequencies. Although it did not recover to the resting hearing level in either group, the diabetic group showed a higher ABR threshold at all ABR frequencies $(4,8,16$, and $32 \mathrm{kHz})$ at 4 weeks after noise exposure, and a higher DPOAE threshold at $8 \mathrm{kHz}$ at 1 week and 2, 3, and 4 weeks, compared with the control group (Table 2, Fig. 2). In order to compensate for the different resting hearing levels before the noise exposure in the two groups, the recovery rates of elevated hearing thresholds after noise exposure were analyzed each week. There were no statistically significant differences in the rate of hearing recovery between the two groups (Table 3 ).

\section{Discussion}

Many studies have reported an association between diabetes and hearing loss [6-12]. Kakarlapudi, et al. [13] reported that sensorineural hearing loss was more common in diabetic patients and that the severity of the hearing loss appeared to correlate with disease progression. A previous animal study reported that diabetic rats had impaired recovery from noiseinduced temporary hearing loss [14]. McQueen, et al. [15], however, found that diabetes mellitus alone did not cause significant capillary changes in the cochlea of a genetic diabetic rats models; the changes were observed only in combination with noise exposure or obesity. Several studies have also reported the possible pathogenesis of diabetes-associated hearing loss $[8,16]$. These reports suggested that this pathogenesis includes cochlear microangiopathy, hyperglycemia of the cerebrospinal fluid or perilymph, auditory neuropathy, diabetic encephalopathy, thickened vessels of the stria vascularis, atrophy of the stria vascularis and loss of outer hair cells.

In our present study in the mouse, the resting hearing levels were significantly worse in the diabetic group than in the normal group. After noise exposure, the hearing thresholds were increased in both the control and diabetic groups, but the changes of the hearing thresholds after exposure were not significantly different between the groups. In contrast to previous studies using diabetic rat model $[14,17]$, the recovery rates of deteriorated hearing thresholds after noise exposure were similar in our diabetic and control mice, suggesting that diabetes had no significant effect on hearing recovery from noise-induced hearing loss. In clinical studies, Ishii, et al. [18] have reported that patients with diabetes are more prone to the development of severe noise-induced hearing loss and Agrawal, et al. [19] also observed significant interactions be- 


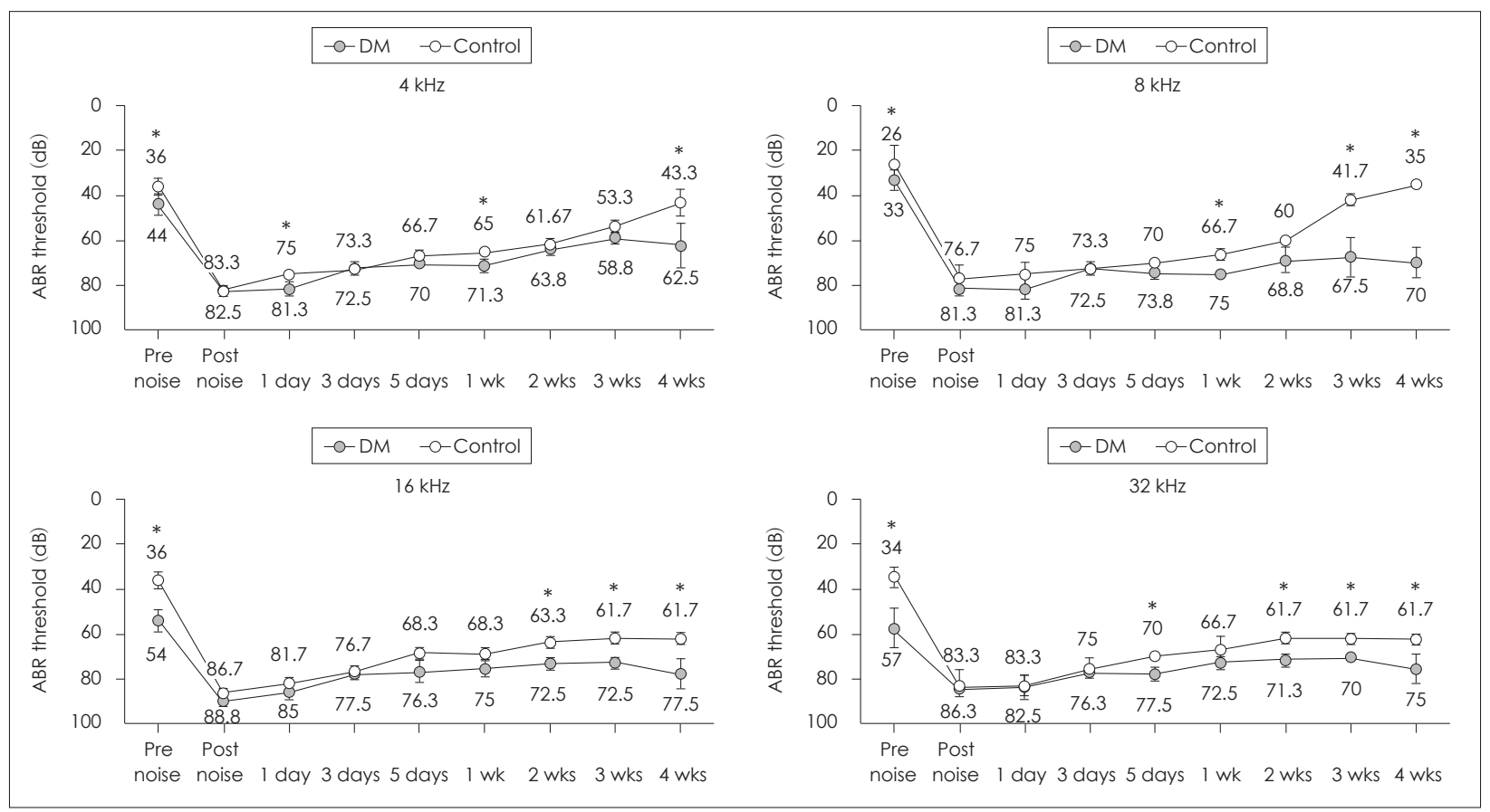

Fig. 2. Thresholds of the auditory brainstem response in control and diabetic mice before and after exposure to noise. The results are reported as the mean $\pm S D$. Mann-Whitney $U$ test. * $p<0.05$ ( $p$-value for the hearing threshold of the control and diabetic groups). DM: diabetes mellitus group, Control: control group.

Table 3. Recovery rates of hearing threshold after noise exposure in the control and diabetic mice

\begin{tabular}{|c|c|c|c|c|c|c|c|c|c|c|c|c|}
\hline \multirow{2}{*}{ Recovery rate (\%) } & \multicolumn{3}{|c|}{$4 \mathrm{kHz}$} & \multicolumn{3}{|c|}{$8 \mathrm{kHz}$} & \multicolumn{3}{|c|}{$16 \mathrm{kHz}$} & \multicolumn{3}{|c|}{$32 \mathrm{kHz}$} \\
\hline & DM & Control & $p$-value* & DM & Control & p-value* & DM & Control & p-value* & DM & Control & p-value* \\
\hline 1 week & $29 \pm 5$ & $39 \pm 4$ & 0.114 & $13 \pm 4$ & $19 \pm 8$ & 0.229 & $40 \pm 9$ & $36 \pm 5$ & 0.400 & $47 \pm 4$ & $32 \pm 19$ & 0.400 \\
\hline 2 weeks & $49 \pm 6$ & $46 \pm 5$ & 0.629 & $26 \pm 11$ & $32 \pm 8$ & 0.400 & $47 \pm 7$ & $46 \pm 5$ & 0.857 & $51 \pm 8$ & $42 \pm 16$ & 0.629 \\
\hline 3 weeks & $62 \pm 6$ & $63 \pm 7$ & 0.629 & $29 \pm 16$ & $68 \pm 10$ & 0.057 & $46 \pm 11$ & $49 \pm 3$ & 1 & $55 \pm 8$ & $43 \pm 6$ & 0.229 \\
\hline 4 weeks & $51 \pm 25$ & $85 \pm 12$ & 0.114 & $23 \pm 14$ & $82 \pm 2$ & 0.057 & $32 \pm 17$ & $49 \pm 3$ & 0.400 & $39 \pm 19$ & $43 \pm 6$ & 0.629 \\
\hline
\end{tabular}

The results are reported as the mean \pm SD. *Mann-Whitney $U$ test ( $p$-value for the recovery rate of the control and diabetic groups). DM: diabetes mellitus group, Control: control group

tween firearm noise exposure and hearing loss in diabetic patients at $3 \mathrm{kHz}$. Previous studies that reported adverse effects of diabetes on noise-induced hearing loss suggested that disrupted microcirculation in diabetic cochlea may cause loss of spiral ganglion cells and irreversible hearing impairment $[14,17]$.

This study had some limitations. First, in our present study with mice, noise-induced hearing impairment was not recovered to the resting hearing level in either the diabetic or control groups. It must be noted that because of the residual hearing impairment at 4 weeks after noise exposure, delayed or limited recovery of diabetic mice may have been missed. We suggested this is the reason why our study showed recovery from noise-induced hearing loss was not affected by diabetes contrary to the previous studies. Therefore, further longterm follow ups of the hearing levels in diabetic mice are needed. Seconds, histopathological studies are needed to as- sess physiological and morphological alterations in the cochleae over time in mice with or without diabetes.

\section{Conclusion}

The hearing level with aging is significantly impaired earlier in diabetic compared with normal mice. After noise exposure, however, the hearing recovery was similar between these groups of mice up to the 4-week follow-up. Further longterm studies of the hearing levels in diabetic mice are needed.

\section{Acknowledgments}

This work was supported by a National Research Foundation of Korea (NRF) grant funded by the Korean government (MEST) (20110026811).

\section{REFERENCES}

1) James S, Gallagher R, Dunbabin J, Perry L. Prevalence of vascular 
complications and factors predictive of their development in young adults with type 1 diabetes: systematic literature review. BMC Res Notes 2014;7:593.

2) Romero P, Salvat M, Fernández J, Baget M, Martinez I. Renal and retinal microangiopathy after 15 years of follow-up study in a sample of Type 1 diabetes mellitus patients. J Diabetes Complications 2007;21:93-100.

3) Ackermann PW, Hart DA. Influence of Comorbidities: Neuropathy, Vasculopathy, and Diabetes on Healing Response Quality. Adv Wound Care (New Rochelle) 2013;2:410-21.

4) Pacher P, Szabó C. Role of poly(ADP-ribose) polymerase-1 activation in the pathogenesis of diabetic complications: endothelial dysfunction, as a common underlying theme. Antioxid Redox Signal 2005;7:1568-80.

5) Makishima K, Tanaka K. Pathological changes of the inner ear and central auditory pathway in diabetics. Ann Otol Rhinol Laryngol 1971;80:218-28.

6) Bainbridge KE, Hoffman HJ, Cowie CC. Diabetes and hearing impairment in the United States: audiometric evidence from the National Health and Nutrition Examination Survey, 1999 to 2004. Ann Intern Med 2008;149:1-10.

7) Mitchell P, Gopinath B, McMahon CM, Rochtchina E, Wang JJ, Boyages SC, et al. Relationship of Type 2 diabetes to the prevalence, incidence and progression of age-related hearing loss. Diabet Med 2009;26:483-8.

8) Austin DF, Konrad-Martin D, Griest S, McMillan GP, McDermott D, Fausti S. Diabetes-related changes in hearing. Laryngoscope 2009; 119:1788-96.

9) Diniz TH, Guida HL. Hearing loss in patients with diabetes mellitus. Braz J Otorhinolaryngol 2009;75:573-8.
10) Rózańska-Kudelska M, Chodynicki S, Kinalska I, Kowalska I. [Hearing loss in patients with diabetes mellitus type II]. Otolaryngol Pol 2002;56:607-10.

11) Celik O, Yalçin S, Celebi H, Oztürk A. Hearing loss in insulin-dependent diabetes mellitus. Auris Nasus Larynx 1996;23:127-32.

12) Cheng YJ, Gregg EW, Saaddine JB, Imperatore G, Zhang X, Albright AL. Three decade change in the prevalence of hearing impairment and its association with diabetes in the United States. Prev Med 2009;49:360-4.

13) Kakarlapudi V, Sawyer R, Staecker H. The effect of diabetes on sensorineural hearing loss. Otol Neurotol 2003;24:382-6.

14) Wu HP, Cheng TJ, Tan CT, Guo YL, Hsu CJ. Diabetes impairs recovery from noise-induced temporary hearing loss. Laryngoscope 2009;119:1190-4.

15) McQueen CT, Baxter A, Smith TL, Raynor E, Yoon SM, Prazma J, et al. Non-insulin-dependent diabetic microangiopathy in the inner ear. J Laryngol Otol 1999;113:13-8.

16) Fukushima H, Cureoglu S, Schachern PA, Paparella MM, Harada T, Oktay MF. Effects of type 2 diabetes mellitus on cochlear structure in humans. Arch Otolaryngol Head Neck Surg 2006;132:934-8.

17) Fujita T, Yamashita D, Katsunuma S, Hasegawa S, Tanimoto H, Nibu K. Increased inner ear susceptibility to noise injury in mice with streptozotocin-induced diabetes. Diabetes 2012;61:2980-6.

18) Ishii EK, Talbott EO, Findlay RC, D’Antonio JA, Kuller LH. Is NIDDM a risk factor for noise-induced hearing loss in an occupationally noise exposed cohort? Sci Total Environ 1992;127:155-65.

19) Agrawal Y, Platz EA, Niparko JK. Risk factors for hearing loss in US adults: data from the National Health and Nutrition Examination Survey, 1999 to 2002. Otol Neurotol 2009;30:139-45. 